\title{
Reflets
}

Revue ontaroise d'intervention sociale et communautaire

\section{La famille-cliente en services de santé : une approche systémique}

\section{Yvonne St-Denis, Elena Popiea et Ginette Coutu-Wakulczyk}

Volume 6, numéro 1, printemps 2000

Approches d'intervention : définir et renouveler nos pratiques

URI : https://id.erudit.org/iderudit/026301ar

DOI : https://doi.org/10.7202/026301ar

Aller au sommaire du numéro

Éditeur(s)

Reflets : Revue ontaroise d'intervention sociale et communautaire

ISSN

1203-4576 (imprimé)

1712-8498 (numérique)

Découvrir la revue

Citer cet article

St-Denis, Y., Popiea, E. \& Coutu-Wakulczyk, G. (2000). La famille-cliente en services de santé : une approche systémique. Reflets, 6(1), 180-190.

https://doi.org/10.7202/026301ar

Tous droits réservés (C) Reflets : Revue ontaroise d'intervention sociale et communautaire, 2000
Ce document est protégé par la loi sur le droit d'auteur. L'utilisation des services d'Érudit (y compris la reproduction) est assujettie à sa politique d'utilisation que vous pouvez consulter en ligne.

https://apropos.erudit.org/fr/usagers/politique-dutilisation/ 


\title{
La famille-cliente en services de santé : une approche systémique
}

\author{
Yvonne St-Denis, \\ inf., B.Sc.N, M.Sc.(cand.), coordonnatrice régionale des services de santé \\ en français, Centre de santé St-Joseph, Sudbury,
}

Elena Popiea,

inf., B.Sc., L.Sc.Ec., M.Sc.(cand.) coordonnatrice d'activités de soins, Centre hospitalier Pierre Janet, Hull et

Ginette Coutu-Wakulczyk,

inf., $P h D$, professeure agrégée, coordonnatrice du programme de maîtrise à distance, Ecole des sciences infirmières, Université d'Ottawa

\section{Quelques repères historiques}

Il est difficile de situer exactement l'origine des soins infirmiers dans l'histoire de l'humanité. Selon Donahue (1996), certaines découvertes archéologiques faites par les anthropologues relient les gestes nourriciers et de soins (caring) à la survie même de la race humaine. Ainsi, au Moyen Âge, la construction des cathédrales, soutenue par des ordres religieux, n'est pas complètement étrangère à l'accomplissement de tels gestes puisque les indigents et les malades se rendaient sur les parvis de l'église pour y obtenir de l'assistance. D'ailleurs, faut-il le souligner, les soins infirmiers dispensés par des religieuses pour soutenir la famille auprès des malades, voire s'y substituer à l'occasion, ont devancé l'avènement de la discipline médicale. Toutefois, il faudra attendre le début de l'ère moderne pour assister à la création de la première école d'enseignement des sciences infirmières, fondée par Florence Nightingale en 1860. 
Celle-ci a ouvert la voie des soins infirmiers à la famille par son travail soutenue auprès des épouses et des enfants de soldats, à qui elle prodiguait enseignement et soutien (Wright et Leahy 1994). Pourtant, Nightingale ne limite pas ses écrits à la seule formation des étudiantes en sciences infirmières. Dans ses Notes on Nursing ${ }^{1}$, Nightingale (1859) a rédigé un guide offrant des conseils à toute femme ayant la responsabilité personnelle de la santé d'autrui. Pour elle, le souci d'inclure la famille dans la prestation de soins est inhérent au travail de l'infirmière.

Ainsi, même dans un contexte de guerre, Nightingale a tenté d'améliorer la santé des militaires en promouvant des habitudes de vie saines et en n'hésitant pas à solliciter la présence d'un membre de la famille auprès des soldats blessés après avoir constaté que leur guérison s'effectuait plus rapidement avec ce genre d'accompagnement. Ces interventions démontrent l'importance qu'elle accordait à la famille dans la prestation des soins (Frey 1993; Stanhope et Lancaster 1988).

De plus, Nightingale ne limite pas ses gestes aux seuls soins immédiats et physiques du patient, mais étend son action bien au-delà, pour y inclure le deuil. En effet, dans une recension des archives, Calkin (1990) rapporte que Nithingale écrivait des lettres aux familles des soldats décédés à Scrutari pour leur transmettre les dernières volontés des défunts. En outre, elle était consciente des besoins de la famille, notamment lorsque la santé de la personne investie du rôle de pourvoyeur était compromise par la maladie, entraînant la famille dans une pauvreté presque certaine à l'époque et mettant en péril son bien-être (Friedman 1998).

"...les premières infirmières reconnaissent le rôle important de la famille dans le maintien de la santé et la guérison de la maladie...»
Ainsi, les premières infirmières reconnaissent le rôle important de la famille dans le maintien de la santé et la guérison de la maladie (Whall 1986). Durant tout le XIX ${ }^{\mathrm{e}}$ siècle, l'infirmière perçoit la famille comme un élément essentiel à la guérison du malade et tient compte de cet environnement global pour le soigner. Le potentiel de connaissances, le milieu physique et les ressources financières de la famille sont alors pris en considération pour éviter la contamination, la contagion et assurer la guérison du malade. 


\section{La famille-cliente : une approche systémique}

"...les dernières

décennies ont été le

témoin d'un

renouveau d'intérêt de

l'infirmière envers la

famille comme

récipiendaire de

soins...”
PourWright et Leahy (1994), même si les soins infirmiers avaient pour objectif de traiter la famille comme un tout, dans les faits, les interventions infirmières ne s'adressaient bien souvent qu'à certains membres de la famille, sans tenir compte de la dynamique familiale dans son ensemble. C'est ce qui fait dire à Gillis (1991) que la famille remplissait alors un rôle utilitaire dans le rétablissement de l'individu malade. La famille sert ici de contexte au malade (famille-contexte). Un tel constat sur la place occupée par la famille n'est pas étranger au développement de la technologie médicale au cours du $\mathrm{XX}^{\mathrm{e}}$ siècle qui a changé considérablement les mentalités et auxquelles les services de soins infirmiers ont dû s'adapter en se centrant sur l'individu, récipiendaire des interventions. Nulle part cette modification estelle aussi apparente que dans le passage des soins prodigués à domicile à ceux effectués en milieu hospitalier. Or, ce passage a eu pour conséquence d'exclure la famille des soins au malade, tout comme il a aussi écarté la famille des événements significatifs tels la mort et la naissance (Wright et Leahy 1994).

Toutefois, les dernières décennies ont été le témoin d'un renouveau d'intérêt de l'infirmière envers la famille comme récipiendaire de soins, d'où l'apparition de la distinction entre la «famille-contexte» et la "famille-cliente» qui suppose une philosophie différente dans la livraison des soins à la famille.Aussi, aujourd'hui, le défi qui anime l'approche de la famille-cliente consiste plus à s'entendre sur une définition de la famille et sur la place qu'il faut lui accorder dans la prestation des soins.

\section{Perspectives théoriques}

Entre les années 1950 et 1990, plusieurs définitions de la famille ont été élaborées. Par exemple, en biologie, la famille est 
déterminée par l'aspect génétique et le réseau biologique entre les personnes. Au niveau social, dans une perspective purement statistique, le Bureau de recensement des États-Unis définit la famille de manière légale comme étant un groupement de deux personnes ou plus dont les membres ont des liens de sang ou sont liés par l'adoption, la garde ou le mariage et qui entretiennent des relations réciproques. Pour leur part, les sociologues définissent la famille comme étant un groupe de personnes vivant ensemble tandis que les psychologues parlent d'un groupe de personnes ayant de forts liens émotionnels (Hanson et Boyd 1996).

Adoptant une vision similaire, les premiers écrits en soins infirmiers de la famille reprennent la définition des sociologues Burgess et Locke (1953) et entrevoient la famille, unie par des liens légaux, à travers les concepts de rôles (couple, parent, fratrie), de communication, d'interactions et de culture. Il faut attendre les années 1985 et la création du Department of Family Nursing at Oregon Health Sciences University, pour parvenir à une définition de la famille qui s'éloigne de celle plus traditionnelle établie en fonction des liens de sang, de mariage ou d'adoption. Cette définition, formulée par Hanson et Boyd (1996), précise que la famille est un système social composé de deux personnes ou plus qui coexistent dans un contexte d'attentes affectives réciproques et de responsabilités mutuelles à durée temporelle. Selon cette définition, la famille se caractérise par un engagement, une prise de décision mutuelle et des objectifs partagés.

\section{De la théorie à la pratique}

Cette définition élargit les paramètres de ce qu'est une famille et ouvre la voie à une approche de la famille-cliente. En effet, la famille renvoie ici à deux personnes ou plus qui dépendent l'une de l'autre pour s'offrir mutuellement un soutien émotionnel, physique et économique. De plus, ce sont les membres qui déterminent eux-mêmes leur appartenance à une famille. Ainsi, selon Lepage et al. (1996), il existe en marge des structures familiales 
"L'infirmière qui intervient auprès d'une personne, a donc tout intérêt à vérifier la composition familiale de cette dernière plutôt que tirer des conclusions erronées à partir des images préconçues..." "...il suffit de regarder les interventions faites auprès de la famille dont un membre est hospitalisé aux soins intensifs ou à une personne âgée vivant dans la communauté, pour reconnaître l'importance de la famille dans la pratique quotidienne de l'infirmière». traditionnelles, monoparentales, homosexuelles et reconstituées, des familles substitutives composées de "personnes» significatives telles les voisins, les collègues de travail ou même, aux yeux de certains individus, un animal de compagnie. L'infirmière qui intervient auprès d'une personne, a donc tout intérêt à vérifier la composition familiale de cette dernière plutôt que tirer des conclusions erronées à partir des images préconçues ou prises pour acquises en fonction de sa culture, son âge ou de son milieu social.

En fait, il ne suffit pas de remplacer le mot «personne» par «famille», comme plusieurs théoriciennes en soins infirmiers l'ont laissé croire, pour adopter une approche de la famille-cliente. Pour ce faire, il faut tenir compte de la complexité du système familial (Murphy 1986), tout en n'oubliant pas que le tout, ici la famille, est plus que la somme de ses parties ou de ses membres (Gillis 1991). Issu de la théorie générale des systèmes, ce dernier principe rappelle la nécessité de préciser les concepts utilisés pour circonscrire la réalité familiale afin de guider adéquatement la pratique des sciences infirmières. Or, selon Gillis (1991), l'absence de définition claire des concepts de famille-cliente et de famillecontexte entraîne une grande confusion et permet plusieurs interprétations. C'est pourquoi le débat autour de ces notions persiste encore aujourd'hui.

Si certains auteurs reconnaissent la pertinence de l'approche de la famille-cliente, qui au-delà des points communs, cherche à rendre compte du caractère unique de toutes familles, d'autres réfutent le réductionnisme provoqué par l'adhésion à un seul cadre de référence. Par exemple, Wright et Leahy (1994) de même que Duhamel (1995) préconisent l'approche systémique de la famille sans toutefois préciser le modèle de soins correspondant à cette approche. D'autres soutiennent que cette approche n'est pas meilleure qu'une autre. En dépit de cette controverse théorique, il suffit de regarder les interventions faites auprès de la famille dont un membre est hospitalisé aux soins intensifs ou à une personne âgée vivant dans la communauté, pour reconnaitre l'importance de la famille dans la pratique quotidienne de l'infirmière. 


\section{La pratique}

Bell et Wright (1990) ont noté des lacunes au sein des programmes de formation. En effet, dans certains programmes de baccalauréat, l'étudiante en sciences infirmières est appelée à travailler en milieu clinique auprès de malades sans jamais avoir abordé le concept de famille-cliente. Ce manque de perspective ralentit considérablement la portée des interventions auprès de la famille et l'avancement des connaissances relatives à son sujet.

Peu importe le milieu de pratique (santé et sécurité au travail, centre hospitalier, communauté), l'infirmière ne transige pas directement avec l'ensemble des membres de la famille. Ce facteur ne facilite pas l'élaboration d'outils pouvant faciliter la collecte des données. En effet, la majorité des instruments de collecte des données est élaborée pour une approche thérapeutique individuelle et l'accès restreint imposé aux membres de la famille pour diverses raisons diminue d'autant les possibilités pour les infirmières d'offrir des interventions à la famille-cliente. C'est pourquoi il faut bien distinguer entre la thérapie familiale et un plan de soins qui lui tient compte d'interventions auprès de la famille-cliente au moment de la planification des services (Duhamel 1995).

Dans une revue de la littérature sur l'intervention familiale, Craft et Willadsen (1991) ont noté la difficulté de différencier la famille-cliente de la famille-contexte. Ils rapportent que, depuis 1984, seulement six interventions infirmières auprès de la famillecliente ont démontré leur efficacité de façon empirique. Cette piètre performance, aux dires de Friedman (1992), s'explique par des carences dans les facteurs suivants: l'expérience et la formation de l'infirmière et sa conception de la famille, la philosophie de l'établissement, de l'agence où travaille l'infirmière et l'appui et les moyens mis en place pour soutenir une approche famillecliente. 


\section{Comment intervenir?}

«En milieu hospitalier, la tendance actuelle favorise une approche individualisée des interventions infirmières dans laquelle la famille ne conserve qu'un rôle utilitaire...»
En milieu hospitalier, la tendance actuelle favorise une approche individualisée des interventions infirmières dans laquelle la famille ne conserve qu'un rôle utilitaire au rétablissement et au maintien de la santé de ses membres. Friedman (1998) a identifié une échelle de soins axés sur la famille. Le niveau de base renvoie à la famillecontexte. C'est à ce niveau que la plupart des théories existantes placent le rôle de la famille. Le second niveau est celui de la perception de la famille comme représentant plus que la somme de ses parties. Et finalement, le dernier niveau comprend l'analyse de l'ensemble des sous-systèmes de la famille (Freidemann 1993; Robinson 1995). À ce dernier niveau, l'infirmière reconnaît l'influence réciproque du malade sur la famille et de cette dernière sur la guérison du malade ainsi que la portée des effets sur le maintien de la santé des membres. Cette conscientisation à la famille-cliente est d'autant plus importante que la formation de l'avenir présage un retour vers des soins axés sur l'unité famille-cliente.

L'intégration de la famille-cliente dans le domaine des soins individualisés en milieu clinique fournira le tremplin à l'élaboration et à la validation d'outils pertinents pour la collecte des données. La pertinence des interventions infirmières en milieu hospitalier est toutefois sujette à la formation des infirmières et à la philosophie et aux politiques des établissements de soins. Pour illustrer ces concepts, nous présentons une histoire de cas et son analyse en comparant les approches de la famille-contexte et de la famille-cliente.

\section{Comparaison de la famille-contexte et de la famille- cliente : une étude de cas}

Un homme âgé de 35 ans, victime d'un accident automobile, arrive à l'urgence. Il présente un traumatisme crânien. N'ayant aucune respiration spontanée, il est intubé et placé sous haute 
surveillance médicale au niveau des signes vitaux. Une sonde intracrânienne est insérée, des sondes gastrique, urinaire et artérielle en plus de plusieurs perfusions intraveineuses, réglées par pompes mécaniques, sont installées. Les pupilles demeurent fixes, mais le tracé de l'encéphalogramme montre la présence d'activité cérébrale. Le pronostic est grave et incertain. Les informations partagées avec la famille doivent être dosées, honnêtes quant à la gravité de la situation tout en nourrissant un espoir réaliste. C'est à ce moment que la famille devient cliente avec ses besoins d'information, d'enseignement, d'adaptation (coping), de prise de décision et d'expression d'émotions.

\begin{abstract}
Famille - contexte
Tout en reconnaissant le besoin de la famille d'être présente au chevet du patient, l'infirmière choisit d'abord de prodiguer ses soins jusqu'à ce qu'elle stabilise le patient et veille à ce que celui-ci soit propre et "présentable».
\end{abstract}

L'infirmière répond aux questions posées par les membres de la famille.

L'infirmière décrit et explique à la famille la nature des traitements prodigués au patient.

L'infirmière informe la famille des autres professionnels qui seront appelés à participer au plan de soins.

L'infirmière reconnait les sentiments de peine, de peur et d'incertitude de la famille suite à l'accident de leur proche et leur laisse un espace d'intimité.

\section{Famille - cliente}

L'infirmière rencontre la famille et s'informe de ce qu'elle connaît déjà au sujet de ce genre de blessures et des données pertinentes quant au système de santé.

L'infirmière encourage les membres de la famille à poser des questions afin de répondre à leurs inquiétudes légitimes.

L'infirmière décrit et explique la nature des traitements mais invite aussi les membres de la famille à participer aux soins du patient en leur enseignant par exemple à accomplir certains soins d'hygiène.

L'infirmière facilite la participation de la famille dans un forum de planification de soins et explique le rôle de chacun des intervenants.

L'infirmière facilite l'expression ouverte et le partage de la peine, la peur et l'incertitude entre les membres de la famille. Elle cherche aussi à connaître les dynamiques de la famille (interactions entre les membres, le regard, le toucher, les gestes de tendresse, et l'absence de ceux-ci). 
Si l'infirmière accompagnait la famille-cliente plutôt que l'individu, elle pourrait mieux orienter ses interventions de prévention secondaire (dépistage) et tertiaire (réadaptation) vers les besoins véritables perçus et exprimés par la famille. Afin de relever le défi d'une pratique fondée sur des évidences de recherche, l'avancement des connaissances en sciences infirmières requiert des données qui décrivent, expliquent et proposent des réponses à des problèmes de santé actuels et potentiels (Feetham1990). La description des interventions permettra ainsi de distinguer clairement l'intervention infirmière auprès de la famille-cliente de celle des autres professionnels, membres de l'équipe multidisciplinaire et de mesurer l'effet synergique de cette approche.

\section{Conclusion}

Le passage de la théorie à la pratique n'est pas toujours évident dans un milieu de travail comme un centre hospitalier où souvent l'infirmière est confrontée à une culture de soins de santé axée davantage vers les économies financières. Pourtant, l'importance de cultiver les rôles d'aidants naturels au sein de la famille a été démontrée maintes fois. Lorsqu'un de ses membres a recours à des soins de santé, une famille ayant développé des habitudes d'auto-soins pourra mieux se débrouiller et resserrer ses liens face à l'épreuve de la maladie qu'une famille contrainte par le système de santé à un rôle utilitaire et d'observateur.

Par ailleurs, en santé communautaire, le milieu le plus propice à l'intervention famille-cliente, l'infirmière doit revoir ses stratégies d'action. La promotion de la santé de la collectivité peut tout aussi bien planifier la santé des familles en fonction des divers problèmes de santé ciblés qu'en fonction du niveau d'apprentissage désiré par la famille. L'approche de la famille-cliente offrira d'autant plus de résultats positifs que plusieurs des déterminants de la santé ne peuvent pas être ignorés dans l'élaboration des programmes. L'approche des soins profondément intégrés au modèle systémique 
"Le travail d'équipe entre les aides infirmières, les infirmières cliniciennes et les professionnels de la communauté, est essentiel pour assurer une continuité de soins...» prend en compte tous les membres d'une famille quelle que soit la composition ou la distance géographique. La famille est alors perçue comme un système interactionnel avec sa dynamique interne, ses relations et sa structure familiale où ce qui affecte un membre affecte tous les autres (Friedman 1998).

Bien que les soins infirmiers à la famille soient conçus dans une perspective théorique de famille-cliente, il reste un travail important à accomplir au plan de la pratique. Le travail d'équipe entre les aides infirmières, les infirmières cliniciennes et les professionnels de la communauté, est essentiel pour assurer une continuité de soins et améliorer la formation des nouvelles infirmières afin qu'ensemble, nous puissions relever les défis auxquels la profession est confrontée à l'aube du $\mathrm{XXI}^{\mathrm{e}}$ siècle.

\section{Bibliographie}

BELL, J.M. et L.M. WRIGHT (1990). «Flaws in Family Nursing Education», The Canadian Nurse/ L'infirmière canadienne, vol. 86, no 6, 28-30.

BUR GESS, E.W. et H.J. LOCKE (1953). The Family : From Institution to Companionship, New York, American Book

CALKIN,J.D. (1990). «Escape into a Higher Order», dans L.M.Wright, W.L.Watson et J.M. Bell, The Cutting Edge of Family Nursing, Calgary, Family Nursing Unit Publications.

CRAFT, M.J. et J.A. WILLADSEN (1990). «Interventions Related to Family», Nursing Clinics of North America, vol. 27, no 2, 517-540.

DOLAN, J.A. (1969). History of Nursing, 12th ed., Toronto, W.B. Saunders Company.

DONAHUE, M. P. (1996). Nursing, The Finest Art : An Illustrated History, 2nd ed., Toronto, Mosby.

DUHAMEL, F. (1995). La santé et la famille : une approche systémique en soins infirmiers, Boucherville, Gaëtan Morin éditeur.

FEETHAM, S.L. (1990). «Conceptual and Methodological Issues in Research of Families», dans L.M.Wright,W.L.Watson et J.M. Bell (sous la dir. de), The Cutting Edge of Family Nursing, Calgary, Family Nursing Unit Publications.

FREIDEMANN, M.L. (1993). "The concept of family nursing», dans G. D. Wegner et R.Alexander (sous la dir. de), Readings in family nursing, Philadelphia, Lippincott, 13-22.

FRIEDMAN, M.M. (1998). Family Nursing: Research, Theory and Practice, 4th ed. Stamford, Connecticut, Appleton \& Lange.

FRIEDMAN, M.M. (1992). Family Nursing : Theory and Practice, 3rd ed. Norwalk, Connecticut, Appleton \& Lange. 
FREY, M.A. (1993). «A Theoretical Perspective of Family and Child Health Derived from King's Conceptual Framework for Nursing: A Deductive Approach to Theory Building», dans S.L. Feetham, S.B. Meister, J.M. Bell et C.L. Gilliss (sous la dir. de), The Nursing of Families : Theory, Research, Education and Practice, London, Sage Publications.

GILLIS, C.L. (1991). «Family Nursing Research, Theory and Practice», Image :Journal of Nursing Scholarship, vol. 23, no 1, 19-22.

GILLIS, C. L., B.L. HIGHLEY, B.M. ROBERTS et I.M. MARTINSON (1989). Toward a Science of Family Nursing, Don Mills, Addison-Wesley Publishing Co., Health Sciences Division, Ontario.

GOUVERNEMENT DE L'ONTARIO (1989). Loi de 1987 sur l'accès à l'information et la protection de la vie privée, Toronto. Imprimeur de la Reine.

HANSON, S. et S. BOYD (1996). Family Health Care Nursing :Theory, Practice, and Research, Philadelphia, F.A. Davis Company.

LEPAGE, M., L.G. ESSIEMBRE et G. COUTU-WAKULCZYK (1996). «Variations sur le thème de la famille», The Canadian Nurse: L'infirmière canadienne, vol 92, no 7, 40-44.

MURPHY, S. (1986). «Family Study and Nursing Research», Image : Journal of Nursing Scholarship, vol. 18, no 4, 170-174.

NEUMAN, B. (1982). The Neuman Systems Model. Norwalk, Connecticut,Appleton-Century-Crofts.

NIGHTINGALE, F. (1859). Notes on Nursing, Harrison and Sons, St. Martin's Lane, Reprinted by J.B. Lippincott Company, Philadelphia.

ROBINSON, C.A. (1998). «Unifying distinctions for nursing research with persons and families», dans M.M. Friedman (sous la dir. de), Family Nursing :Theory and Practice, $4^{\text {th }}$ Edition. Norwalk, Connecticut, Appleton \& Lange.

STANHOPE, M. et J. LANCASTER (1988). Community Health Nursing: Process and Practice for Promoting Health. 2nd ed., Toronto, C.V. Mosby Company.

WHALL, A.L. (1986). «The family as the unit of care in Nursing: A historical review», Public Health Nursing, vol. 3, no 4, 240-249.

WRIGHT, L.M. et M.LEAHY (1994). Nurses and Families: A guide to Family Assessment and Intervention, Philadelphia, F.A. Davis.

\section{Note}

1. Pour Nightingale, la guerre de Crimée a non seulement permis les premières compilations statistiques sur les services de santé offerts aux militaires de l'hôpital de campagne de Scrutari, mais a aussi ouvert un nouveau volet de formation en sciences infirmières. 